\title{
Unit cell structure of the wurtzite phase of GaP nanowires: X-ray diffraction studies and density functional theory calculations
}

\author{
Dominik Kriegner, ${ }^{1,}{ }^{*}$ Simone Assali, ${ }^{2}$ Abderrezak Belabbes, ${ }^{3}$ Tanja Etzelstorfer, ${ }^{1}$ Václav Holý, ${ }^{4}$ Tobias Schülli, ${ }^{5}$ \\ Friedhelm Bechstedt, ${ }^{3}$ Erik P. A. M. Bakkers, ${ }^{2}$ Günther Bauer, ${ }^{1}$ and Julian Stangl ${ }^{1}$ \\ ${ }^{1}$ Institute of Semiconductor and Solid State Physics, Johannes Kepler University Linz, Altenbergerstrasse 69, A-4040 Linz, Austria \\ ${ }^{2}$ Department of Applied Physics, Eindhoven University of Technology, $5600 \mathrm{MB}$ Eindhoven, The Netherlands \\ ${ }^{3}$ Institut für Festkörpertheorie und -optik, Friedrich Schiller Universität, 07743 Jena, Germany \\ ${ }^{4}$ Department of Condensed Matter Physics, Faculty of Mathematics and Physics, Charles University, Ke Karlovu 5 , \\ CZ-121 16 Prague 2, Czech Republic \\ ${ }^{5}$ European Synchrotron Radiation Facility, 6 Rue Jules Horowitz, BP 220, 38043 Grenoble Cedex 9, France
}

(Received 2 July 2013; revised manuscript received 23 August 2013; published 30 September 2013)

\begin{abstract}
We present structural characterization of the wurtzite crystal structure of GaP nanowires, which were recently shown to have a direct electronic band gap. The structural parameters of the wurtzite phase do consist of two lattice parameters and one internal degree of freedom, determining the Ga-P bond length along the $c$ direction. Using density functional theory calculations, we study the influence of the internal degree of freedom on the band structure. By synchrotron $\mathrm{x}$-ray diffraction studies near the Ga- $K$ edge we determine the lattice parameters $a=3.8419 \AA$ and $c=6.3353 \AA$ as well as the internal degree of freedom $u=0.37385$ with high accuracy. We find that different Ga-P bond lengths are not equal, in contrast to the case in the zinc blende bulk phase. As a result, a spontaneous polarization is predicted for wurtzite $\mathrm{GaP}$.
\end{abstract}

DOI: 10.1103/PhysRevB.88.115315

PACS number(s): 61.05.C-, 81.07.Gf, 71.15.Mb

\section{INTRODUCTION}

III-V semiconductors are an intensively studied group of materials due to their favorable optical and electronic properties with widespread applications. Apart from the nitrides, the cubic zinc blende (ZB) phase is the stable phase of III-V compound semiconductors under ambient conditions. Hexagonal polymorphs are stable for several III-V compounds only as high-pressure phases. ${ }^{1,2}$ With the recent progress in nanowire growth, however, it became possible to fabricate hexagonal polytypes of several III-V compounds. ${ }^{3}$ The reason for this phenomenon is the fact that nanowires grow very often along the cubic [111] direction, which corresponds to an ABCABC-type stacking of III-V bilayers. In contrast to bulk material, in thin nanowires, the stacking sequence changes easily, and under particular growth conditions can be altered completely to the wurtzite (WZ) crystal structure with an ABABAB-type stacking. ${ }^{4}$ Upon the change of the crystal symmetry, many important material parameters such as the band gap and band alignment are also modified. For most III-V semiconductors such as InP, InAs, GaAs, or InSb, changes of the band gap were found, ${ }^{5-10}$ with the general tendency of a slightly larger direct band gap in the WZ crystal structure as compared to the ZB counterpart. A particularly interesting case is $\mathrm{GaP}$, which is the only $(\mathrm{Ga}, \mathrm{In})-\mathrm{V}$ semiconductor with an indirect gap in the bulk phase. In the wurtzite polytype, the band structure actually exhibits a direct gap, which was predicted theoretically ${ }^{8,11}$ and recently shown experimentally: Wurtzite GaP has a direct band gap of $2.1 \mathrm{eV}$, i. e., in the

Published by the American Physical Society under the terms of the Creative Commons Attribution 3.0 License. Further distribution of this work must maintain attribution to the author(s) and the published article's title, journal citation, and DOI. visible spectrum, which can be tuned over a wider range from 1.8 to $2.25 \mathrm{eV}$ by alloying with $\mathrm{Al}$ and As. ${ }^{12} \mathrm{~A}$ similar change of the band structure was found for another III-V compound with indirect band gap in the bulk ZB phase, namely AlAs. ${ }^{13}$ This is attractive for optoelectronic applications, in particular since $\mathrm{GaP}$ has a negligible lattice mismatch with respect to $\mathrm{Si}$, making it a perfect candidate for integration of optoelectronic elements with mature Si technology. ${ }^{14}$

In order to be able to understand the electronic structure in detail, it is important to know exactly the crystal lattice structure, which means that not only do the lattice parameters have to be determined, but also the exact positions of all atoms within the unit cell need to be known. The WZ crystal structure $\left(P 6_{3} m c\right)$ formed by binary compounds $\mathrm{AB}$ has, besides the lattice parameters $a$ and $c$, one additional free internal parameter $u$ as depicted in Fig. 1(a), which specifies the bond length of the $\mathrm{A}$ and $\mathrm{B}$ atoms within one III-V bilayer. The lateral position of the atom $\mathrm{B}$ in the unit cell is fixed by the symmetry, but the vertical position is a priori unknown. This position is usually specified as a fraction $u$ of the lattice parameter $c$. In an idealized hexagonal configuration, where all bond lengths and bond angles are equal, this internal cell parameter $u$ takes the value of $3 / 8$. In this ideal configuration the environment of all atoms is perfectly tetrahedral and the bond angles $\alpha$ and $\beta$ depicted in Fig. 1(a) correspond to the ideal tetrahedral angle of $109.47^{\circ}$; the ratio of $c / a$ takes the value of $\sqrt{8 / 3}$. The influence of the deviations from the ideal bonding tetrahedrons, in particular of $u$, on the electronic properties of wurtzite materials is hardly understood. Accompanying the symmetry reduction from $\mathrm{ZB}$ to the $\mathrm{WZ}$ structure it is possible that a spontaneous polarization field ${ }^{15,16}$ in the presence of surfaces or interfaces ${ }^{17}$ occurs. The strength of the corresponding internal electric field depends on the bond ionicity and the hexagonal crystal field. 

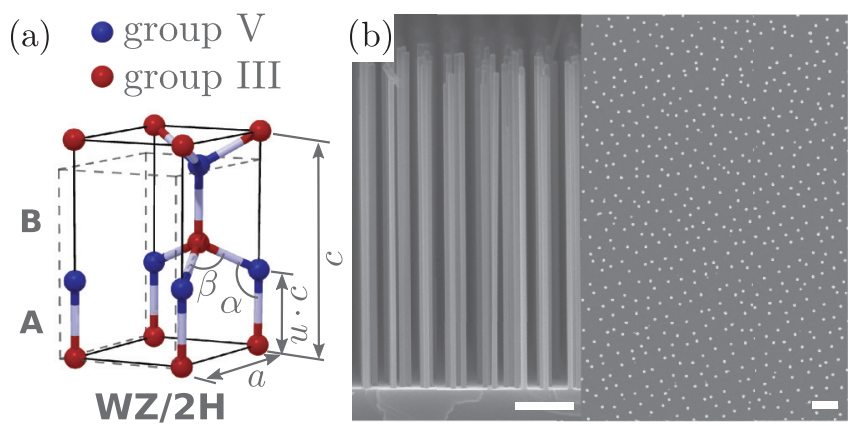

FIG. 1. (Color online) Panel (a) shows a sketch of the WZ unit cell with stacking sequence ABAB $\cdots$ of III-V bilayers. Shown are the lattice parameters $a$ and $c$ as well as the internal parameter $u$, specifying the bond length along the $c$ direction within the III-V bilayer, and the bond angles $\alpha$ and $\beta$. Also indicated, however, exaggerated, is the deformation of the unit cell with respect to what would be expected from bulk ZB bond lengths. In panel (b) scanning electron micrographs recorded in side view (left) and top view (right) are shown. The homogeneous length and areal density of the nanowires can be seen. The scale bars correspond to $1 \mu \mathrm{m}$.

The sign and the strength of this electric field are characterized by the deviation $\varepsilon=(u-3 / 8)$ of the actual $u$ parameter from its ideal value. ${ }^{16}$

In this work we report on the determination of the detailed crystal structure of GaP WZ nanowires by synchrotron x-ray diffraction (XRD). So far such measurements of the internal structure of similar compounds were mostly done on powders using the Rietveld method ${ }^{2,18}$ or bulk crystals ${ }^{19-21}$ or deduced from theoretical calculations. , 21,22 We use highly intense synchrotron radiation to enable the measurement also on the nanometer-sized crystallites of an ensemble of GaP nanowires. Such a measurement requires the investigation of not only the Bragg peak positions, but also of their intensity, since it is the intensity of the Bragg reflections which holds the information about the internal structure of the unit cell. The evaluation of just the Bragg peak positions yields only the lattice parameters $a$ and $c$. By exploiting the energy tunability of the synchrotron source we increase the sensitivity of the diffracted intensities with respect to the internal parameter in order to make its determination possible with high enough precision.

\section{METHODS AND EXPERIMENTS}

To determine the internal structure of the unit cell, the diffracted intensity of a set of WZ Bragg reflections needs to be compared. The $\mathrm{WZ}$ phase in $\mathrm{GaP}$ has only been realized in the form of nanowires. In our case they are grown by the vapor-liquid-solid (VLS) mechanism by metal-organic vapor phase epitaxy on GaP (111)B substrates using gold nanoparticles as catalysts. ${ }^{12}$ For the diffraction measurements we require a macroscopic area on the sample with homogeneous density and length of nanowires in order to obtain comparable diffracted intensities for measurements at different Bragg reflections, where slightly different parts of the sample contribute to the diffracted signal. When the nanowires are grown on a prepatterned substrate the local fluctuations of the density and of the growth conditions are minimized, resulting in a homogeneous ensemble of nanowires. $100 \mathrm{~nm}$ large gold particles were deposited using the nanoimprint technique in a square pattern with a $500 \mathrm{~nm}$ pitch on $\sim 1 \mathrm{~cm}^{2}$ large substrates. From such an ordered pattern of gold particles an ensemble of nanowires with homogeneous density and length of 5.4 to $5.8 \mu \mathrm{m}$ evolves during growth. The length of the nanowires fluctuates from wire to wire in the given range; the average length of the ensemble shows, however, no measurable change over distances of $200 \mu \mathrm{m}$. On extended length scales of $2 \mathrm{~mm}$ we find a variation of the average length of $\sim 4 \%$. Figure 1(b) shows a scanning electron microscopy (SEM) image of our sample. Further growth details can be found in Ref. 12. Transmission electron microscopy (TEM) investigations showed that the nanowires crystallize in the WZ crystal structure with a stacking fault density of less than 1 per $\mu \mathrm{m}$ length. The low stacking fault density found by TEM, where only a chosen few nanowires can be investigated, was also confirmed by our XRD measurements on the nanowire ensemble. ${ }^{12}$ These measurements show narrow peaks (full widths at half maximum of 0.0015 to $0.0026 \AA^{-1}$ ) along the [000.1] direction, corresponding to long defect-free segments within the nanowires. ${ }^{23}$ A low stacking fault density is also required for the determination of the internal parameter since stacking faults would lead to a broadening of the Bragg peaks, which at some point renders the determination of the peak intensity impossible.

X-ray diffraction measurements were performed at beamline ID01 of the European Synchrotron Radiation Facility (ESRF), Grenoble using a monochromatic x-ray beam of $\sim 100 \times 100 \mu \mathrm{m}^{2}$ size and an area detector. A sketch of the coplanar diffraction geometry is shown in Fig. 2(a). Measurements were performed at the set of $\left(10 \overline{1} \cdot \bar{l}_{1}\right)$ and $\left(20 \overline{2} . \bar{l}_{2}\right)$ Bragg reflections. Those reflections are aligned along [000. $\overline{1}$ ] crystal truncation rods. Only certain $l_{1,2}$ values are accessible in Bragg geometry, were a transmission through the sample [Laue zone in Fig. 2(a)] is not possible due to absorption in the bulk substrate. At the used x-ray energies and within the restrictions of the used goniometer, in total nine such Bragg reflections are available $\left(l_{1}=5, \ldots, 9, l_{2}=6, \ldots, 9\right)$ covering scattering angles from $\sim 61^{\circ}$ to $136^{\circ}$. The orientation
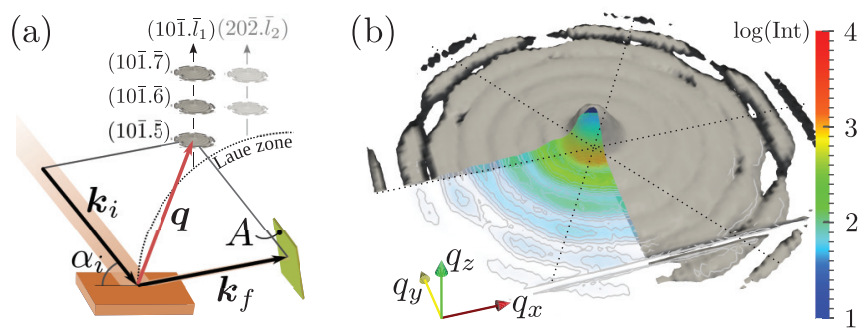

FIG. 2. (Color online) Panel (a) shows a sketch of the diffraction setup with the primary and diffracted wave vectors $\boldsymbol{k}_{i, f}$ as well as their difference the momentum transfer $\boldsymbol{q}$. The detector area $A$, which is perpendicular to the diffracted wave vector is indicated by a plane marked in green color. The diffraction positions of some $(h 0 \bar{h} . \bar{l})$ Bragg positions along [000.1] crystal truncation rods are illustrated. In panel (b) a three-dimensional contour plot of the measured intensity pattern of the (101. $\overline{5}) \mathrm{WZ}$ Bragg reflection is shown. The contour plot is cut open to show the intensity variation inside as a semitransparent color plot. Dotted lines indicate the facet streaks due to the hexagonal cross section of the nanowires. 
of the nanowire lattice is given by the substrate, which is (111)B, leading to the fact that only Bragg peaks with negative $l$ can be observed in Bragg geometry. X-ray energies of 10257 and $10305 \mathrm{eV}$ well below the Ga absorption edge at $10368 \mathrm{eV}$ were chosen to avoid fluorescence background. By rocking the sample [scanning the incidence angle $\alpha_{i}$ shown in Fig. 2(a)] in the vicinity of these Bragg peaks, threedimensional (3D) reciprocal space maps as the one shown in Fig. 2(b) were recorded. The measurements show several orders of size oscillations, which indicate the uniformity of the nanowire thickness within the ensemble. At every x-ray energy two distinct spots on the sample were used for the measurements, which means that in total four independent data sets have been recorded. Each data set consists of measurements at all of the mentioned Bragg diffractions, i. e., nine three-dimensional reciprocal space maps. Analyzing the Bragg peak positions yields the lattice parameters for $\mathrm{WZ} \mathrm{GaP}$ of $a=3.8419 \pm 0.0004 \AA$ and $c=6.3353 \pm 0.0005 \AA$. The same lattice parameters were extracted from laboratory XRD experiments of a series of samples grown on prepatterned as well as unpatterned substrates with areal density of nanowires ranging from 0.003 to 4 per $\mu \mathrm{m}^{2}$ as well as varying diameters from $\sim 50$ to $\sim 100 \mathrm{~nm}$.

For the analysis of the intensities the kinematical diffraction theory ${ }^{24}$ was used. Within the kinematical theory the diffracted intensity is proportional to the squared absolute value of the structure factor, effects of absorption and extinction are neglected. We will discuss the applicability of this approximation below. The internal parameter enters in the structure factor of the unit cell and therefore the diffracted intensities are sensitive to this parameter. For the $\mathrm{WZ}$ phase the structure factor is given by

$$
\begin{aligned}
F_{\text {cell }}(\boldsymbol{q}(h, k, l), E)= & \left(f_{\mathrm{Ga}} e^{-\frac{B_{G a}}{16 \pi^{2}} q^{2}}+f_{\mathrm{P}} e^{-\frac{B_{P}}{16 \pi^{2}} q^{2}} e^{i 2 \pi u l}\right) \\
& \times\left\{1+\exp \left[i 2 \pi\left(\frac{h+2 k}{3}+\frac{l}{2}\right)\right]\right\}
\end{aligned}
$$

with the energy and momentum transfer dependent atomic scattering factors $f_{\mathrm{Ga}, \mathrm{P}}=f(q, E)$, taken from Ref. 25, and the isotropic Debye-Waller exponents $B_{\mathrm{Ga}, \mathrm{P}}$ are considered separately for the $\mathrm{Ga}$ and $\mathrm{P}$ atoms. ${ }^{26}$ The Debye-Waller factors are used to describe the decrease of the diffracted intensity at higher momentum transfers due to the thermal oscillation of the atoms around their rest positions. Therefore they are temperature dependent. The momentum transfer $\boldsymbol{q}$ of the scattering process is given by the difference of the diffracted and incident wave vectors $\left(\boldsymbol{q}=\boldsymbol{k}_{f}-\boldsymbol{k}_{i}\right)$ as sketched in Fig. 2(a). To determine the optimal conditions for the measurement of the internal parameter the variation of the kinematic diffracted intensity was investigated as a function of energy and internal parameter. Instead of $u$ it is more convenient to express it as the deviation from its ideal value $\varepsilon=u-3 / 8$. A plot showing the variation of the intensity vs $\varepsilon$ in the region of the Ga- $K$ absorption edge is depicted in Fig. 3(b). Different Bragg peaks show a different variation of the intensity with the internal parameter as outlined by Eq. (1). Compared to measurements with $\mathrm{Cu} K \alpha$ radiation, using $\mathrm{X}$-ray energies close to the $\mathrm{Ga}$ absorption edge results in an enhancement of the sensitivity of the diffracted intensities with respect to the internal parameter by more than a factor

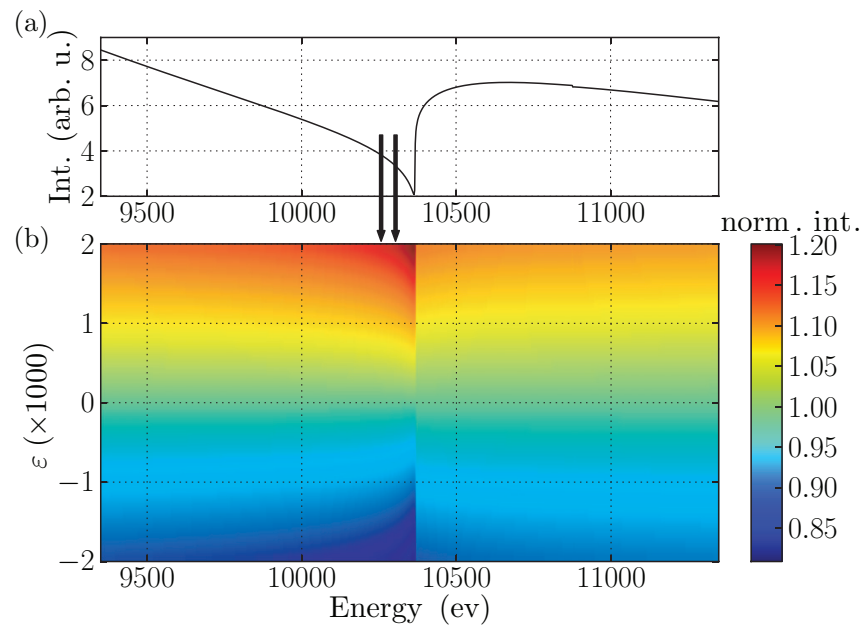

FIG. 3. (Color online) Variation of the diffracted intensity of the

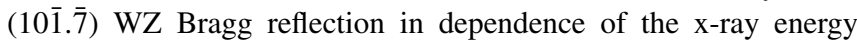
and the internal parameter around the Ga-K absorption edge. Panel (a) shows the variation of the total intensity (for $\varepsilon=0$ ) vs the $\mathrm{x}$-ray energy, and panel (b) shows the variation of the normalized intensity with respect to the internal parameter $\varepsilon$. In the vicinity of the absorption edge the diffracted intensity is most sensitive to the internal parameter. Arrows indicate the two energies used in the experiment.

of 2. However, even in the optimal case for an energy at the absorption edge only a $20 \%$ intensity change is expected for the $(h 0 \bar{h} . \overline{7})$ Bragg peaks in the plotted $\varepsilon$ range. Together with the $(h 0 \bar{h} . \overline{9})$ peaks the $(h 0 \bar{h} . \overline{7})$ Bragg peaks are the most sensitive ones to detect a change in the internal parameter. The absolute intensity has, however, a minimum for energies close to the $\mathrm{Ga}$ absorption edge as can be seen in Fig. 3(a). This outlines the necessity of using a highly intense energy tunable $\mathrm{X}$-ray source as provided by a synchrotron in order to measure the Bragg diffraction intensity with high enough fidelity.

Furthermore, also the shape function $S$, i. e., the Fourier transformation of the nanowires shape, which depends on the nanowire diameter $D$ (distance of two opposite lying facets) and length $L$, enters in the calculation of the intensity to be expected at a certain detector position $\boldsymbol{r}$, giving the direction of the exit wave vector $\boldsymbol{k}_{f}$.

The diffracted intensity at a certain reciprocal lattice point $\boldsymbol{h}$ is given by

$$
I(\boldsymbol{r}, \boldsymbol{h})=I_{0} \frac{C}{r^{2}}\left|F_{\text {cell }}(\boldsymbol{h}) S(\boldsymbol{q}(\boldsymbol{r})-\boldsymbol{h}, D, L)\right|^{2}
$$

with the primary beam intensity $I_{0}$ and a constant $C=$ $\left(|\boldsymbol{k}|^{4} \lambda^{4} r_{\mathrm{el}}^{2}\right) /\left(16 \pi^{4} V_{\text {cell }}^{2}\right)$ depending on the classical electron radius $r_{\mathrm{el}}$ and the volume of the WZ unit cell $V_{\text {cell }}$. Equation (2) is valid for diffraction experiments performed with $s$-polarized x-ray photons, i. e., polarization direction perpendicular to the scattering plane. The shape function of the hexagonal nanowires was calculated analytically in Ref. 27 . It is this shape function which determines the shape of the diffraction peaks observed in the three-dimensional reciprocal space map shown in Fig. 2(b). The hexagonal shape of the wires can be seen from the sixfold symmetry of the diffracted signal, which is indicated by dotted lines. Although the measurement is performed on an ensemble of nanowires, 
the small size distribution makes the observation of several orders of intensity oscillations possible. To obtain comparable intensities at different Bragg peaks we furthermore need to consider that a different number $N$ of wires are illuminated at the different Bragg diffraction spots due to different incidence angles with respect to the sample surface. In order to model exactly the measured intensity we integrate over the detector area $A$, i. e., the area spanned by the array of pixels of the 2D detector, which is a plane in real space as shown in Fig. 2(a). The integration is, however, performed in reciprocal space, where this plane corresponds to a segment of a sphere (Ewald's sphere) since it is always locally perpendicular to the diffracted wave vector. This means that the final observable intensity in the detector area at an incidence angle $\alpha_{i}$ is given by

$$
I_{\text {total }}\left(\alpha_{i}, \boldsymbol{h}\right)=\int_{A} d^{2} r N\left(\alpha_{i}\right) I(\boldsymbol{r}, \boldsymbol{h}) .
$$

In general this integration can not be performed analytically due to the complicated intensity distribution and nontrivial integration geometry. Therefore, numerical integration based on the double quadrature is used.

We use this formalism in order to describe the summed intensity of the area detector during a sample rocking scan. Doing so we avoid any numerical errors resulting from data treatment (gridding of the data) during conversion to reciprocal space. We rather calculate the intensities in angular space and from that the integrated intensity on the detector, which we can directly compare to the experimental curves as shown in Fig. 4.

Equation (3) describes the intensity for a perfectly monodisperse ensemble of nanowires producing highly visible size fringes (dashed line in Fig. 4) which are not observed in the experimental data. Considering Gaussian distributions of the

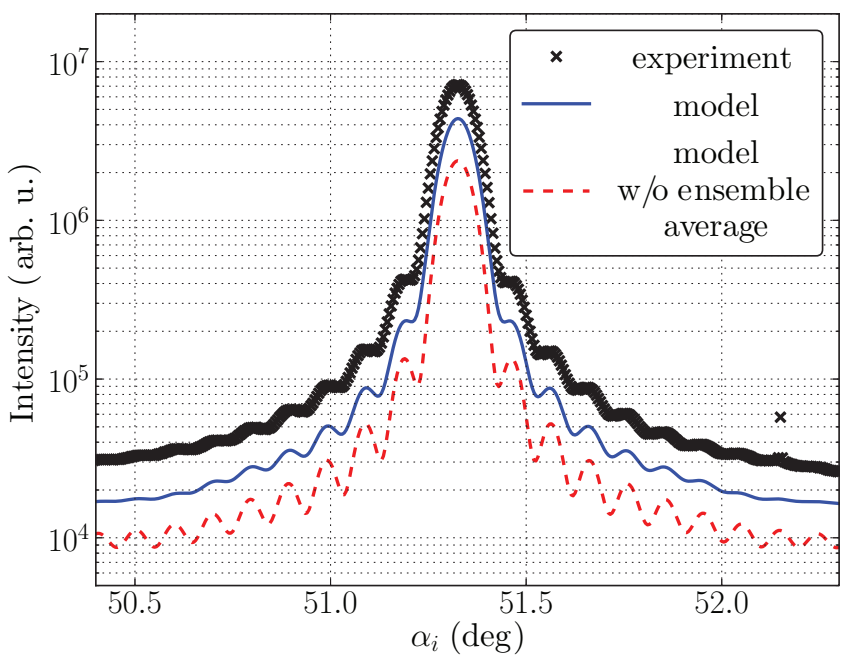

FIG. 4. (Color online) Integrated detector signal during a sample x-ray rocking scan at the $(10 \overline{1} . \overline{5})$ Bragg reflection of GaP. For comparison two different simulations of the intensity are shown, one depicting the modeled detector signal for a perfectly monodisperse ensemble of nanowires (dashed red line) and one showing the simulated signal including ensemble averaging (solid blue line), which accounts for a Gaussian size distribution of $80 \pm 3 \mathrm{~nm}$ with and tilt distribution with sigma $\sigma=0.016^{\circ}$ of the nanowires. The curves are shifted vertically for clarity. nanowire diameters as well as their orientation distribution reproduces the measured data. The orientation distribution of the nanowires describes the fact that the nanowires are not perfectly oriented with respect to each other, which effectively produces a distribution of incidence angles. While the variation of the incidence angle smears out the size fringes uniformly across the spectrum, the diameter variation has more influence further away from the central diffraction peak. Hence both effects can be disentangled, and we find an average diameter $D_{0}=80 \mathrm{~nm}$ with a distribution width $\sigma_{D}=3 \mathrm{~nm}$, and a orientation distribution around the surface normal with a width of $\sigma_{O}=0.016^{\circ}$.

From the scaling factors necessary to obtain the experimentally observed intensities we determine the experimental structure factors $F_{\exp }$ of the $\left(10 \overline{1} . \bar{l}_{1}\right)$ and $\left(20 \overline{2} . \bar{l}_{2}\right)$ Bragg reflections. These values are fitted to the model of the structure factor of the unit cell given in Eq. (1) by minimizing the weighted $R$ value ${ }^{28}$

$$
R_{w}=\left(\frac{\sum w\left|F_{\exp }-F_{\text {cell }}\right|^{2}}{\sum w F_{\exp }^{2}}\right)^{1 / 2} .
$$

As parameters for the optimization the internal parameter and the two Debye-Waller exponents were used. The weights were chosen to be $w=1 /\left(0.01 F_{\text {exp }}\right)$. Using the counting statistics one would obtain much smaller errors for the measured structure factors; however, repeating the same measurement at the same spot and energy we found values differing by approximately $1 \%$. Most probably the repeatability is limited by the quality of the monitor signal needed to correct for fluctuations of the primary beam intensity. From the fit we obtain $\varepsilon=-(115 \pm 17) \times 10^{-5}(u=0.37385 \pm 0.00017)$ and $B_{\mathrm{Ga}}=0.53 \pm 0.05 \AA^{2}$ and $B_{\mathrm{P}}=0.66 \pm 0.02 \AA^{2}$ with an average $R_{w}$ value of $0.47 \% .^{29}$ The fitted values for the internal parameter were first determined independently for the four recorded datasets measured at different positions on the sample and using two different X-ray energies of 10257 and $10305 \mathrm{eV}$. We find values ranging from $-134 \times 10^{-5}$ to $-88 \times 10^{-5}$ for $\varepsilon$. Standard deviations listed above are obtained from averaging the values obtained from the four independent measurements. The $B$ values, fitted simultaneously with the internal parameter, for our data measured at room temperature (297 K), correspond quite well to the values found for the $\mathrm{Ga}$ and $\mathrm{P}$ atoms in the $\mathrm{ZB}$ bulk phase. ${ }^{26}$

As outlined above we use the kinematical approximation to describe the diffracted intensities. Within this approximation the absorption and the extinction are neglected. The absorption length of $\mathrm{WZ} \mathrm{GaP}$ at the used x-ray energies is above $78 \mu \mathrm{m}$ and therefore much larger than the path of the x-rays in the material of the nanowires, which is always below a few microns. Also the extinction length, which depends on the strength of the particular reflection, is much larger than the average x-ray path length. However, comparing the extinction length of the by far strongest Bragg reflection, the $(10 \overline{1} . \overline{5})$ reflection, which is a factor of 7 stronger than any other measured peak, we find that for this reflection the extinction might give an effect slightly larger than the error of the measurements which is therefore no longer fully negligible. This is also seen in the fit of our data. When we include the $(10 \overline{1} . \overline{5})$ Bragg peaks in our data analysis 
we find that the average $R_{w}$ value increases significantly to $1.57 \%$, although we still find a similar internal parameter $\left[\varepsilon=-(111 \pm 15) \times 10^{-5}\right]$. Thus for our sample geometry and when excluding the strong $(10 \overline{1} . \overline{5})$ Bragg peak, the kinematical approximation is fulfilled with high precision.

\section{THEORY}

In the following the results of the structural investigations are compared with results of density functional theory (DFT) calculations performed within the local density approximation (LDA) for exchange and correlation. ${ }^{30}$ Details of the method are described in Refs. 8,16. The values of the three structutral parameters $a, c$, and $\varepsilon$ are derived by minimization of the total energy as a function of those parameters. We apply a three-step procedure: For initially zero $\varepsilon$ the total energy $E=E(a, c)$ is determined on a regular $(a, c)$ grid. Then the cell-internal parameter $\varepsilon$ is optimized with respect to the total energy for the optimal $(a, c)$ determined in the previous step. In the last step $E(a, c)$ is minimized again, but now under the constraint of the obtained cell-internal parameter. The resulting energy surface is illustrated in Fig. 5. Along with the optimal lattice parameters a $c / a$ ratio larger than $\sqrt{8 / 3}$ is found. Within the last step we resolve the interplay of unit cell shape $(a, c)$ and the cell-internal relaxation, at least in a first-order manner. The lattice constants and the internal parameter are determined if the Hellmann-Feynman forces are smaller than $1 \mathrm{meV} / \AA$. Compared to simultaneous optimization of all structural parameters our method provides sufficient accuracy while dramatically reducing the computational effort.

As a consequence of the overbinding tendency in LDA the resulting lattice constants, determined from the minimum of the total energy in Fig. 5, are smaller than the experimental ones but their relative changes from $\mathrm{ZB}$ to $\mathrm{WZ}$ are in reasonable agreement with the experimental findings, similar to findings for other III-V compounds. ${ }^{31,32}$ Due to the small absolute value of $\varepsilon=(u-3 / 8)$ its precise determination is

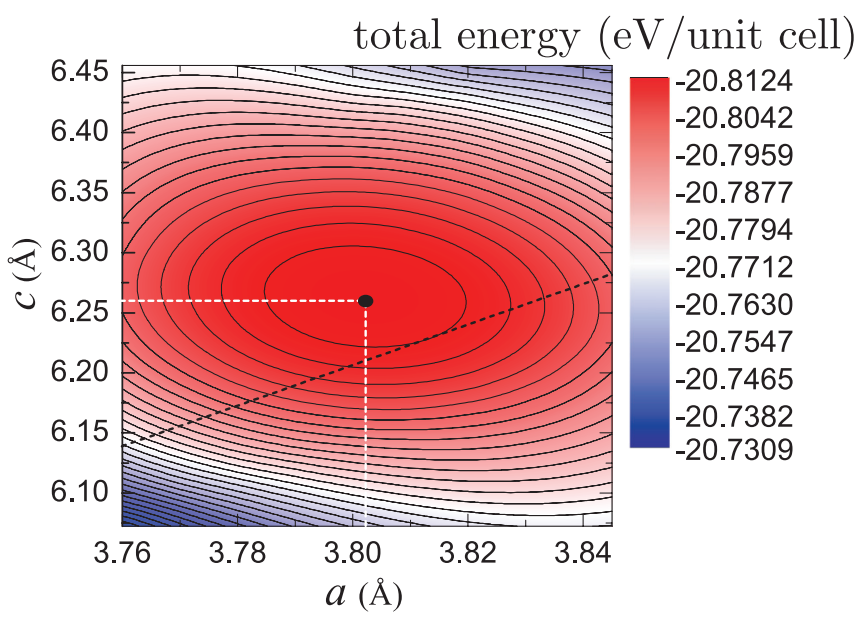

FIG. 5. (Color online) Total energy per unit cell determined by DFT calculations in dependence of the lattice paramters $(a, c)$. From the minimum in the energy surface (marked by a black dot) the optimal lattice parameters are determined. The optimized lattice parameter clearly show a $c / a$ ratio larger than $\sqrt{8 / 3}$, which is indicated by a dashed line.

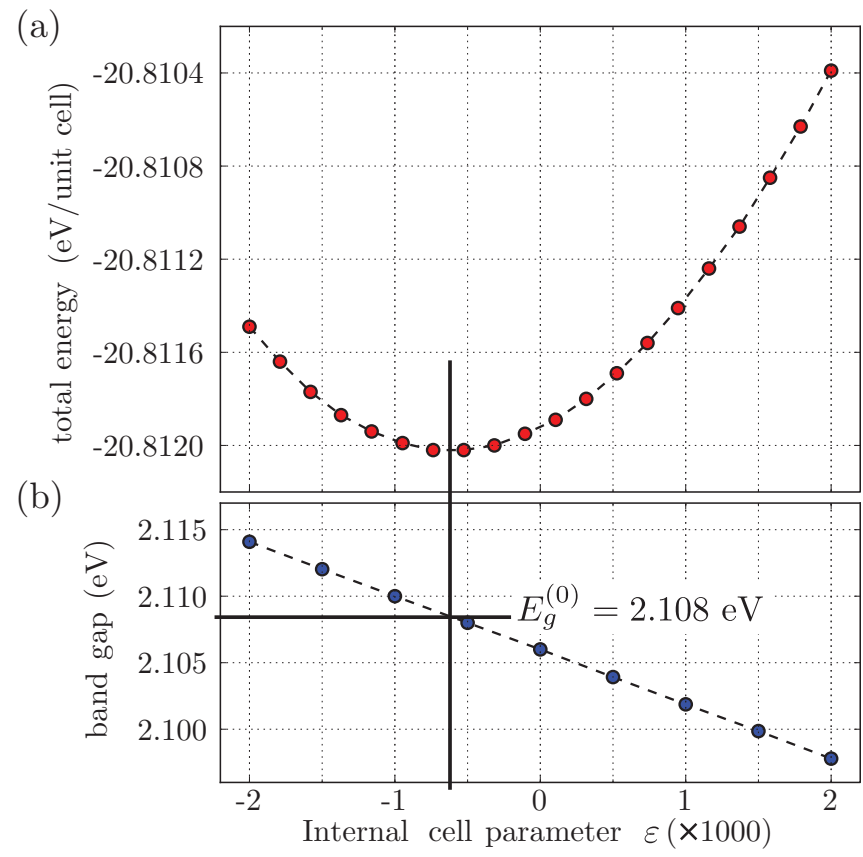

FIG. 6. (Color online) Influence of the internal cell parameter $\varepsilon$ on the total energy per unit cell (a) and fundamental energy gap (b) derived from DFT calculations. The equilibrium band gap $E_{g}^{(0)}$ at the minimum of the total energy is indicated.

difficult: By the minimization of the total energy we find $\varepsilon=$ $-6 \times 10^{-4}$. To check this result we performed complementary calculations within the generalized gradient approximation (GGA) for exchange and correlation. It is well known that LDA underestimated the equilibrium volume, while the GGA slightly overestimates it. This is also represented by the lattice parameters we find using these two approximations. However, the LDA gives in general better results for the structural parameters of conventional III-V compounds in comparison with experimental data. ${ }^{33}$ Although the GGA method determines a lattice parameter larger then the experimental ones, the internal parameter we find is still negative: We obtain a value of $\varepsilon=-7 \times 10^{-4}$. The value for $\varepsilon$ computed for bulk wurtzite does therefore only marginally depend on the used exchange-correlation functional, is clearly negative, and agrees within a factor of 2 with the value derived from XRD measurements of nanowires.

An illustration of the importance of the internal cell parameter $\varepsilon$ is given in Fig. 6. It shows that the total energy possesses a pronounced minimum around the equilibrium value although the energy changes are small. The minimum corresponds to the equilibrium value of $\varepsilon$ as discussed above. The complete band structure showing the directness of the wurtzite-GaP semiconductor can be found elsewhere. ${ }^{8}$ The influence of the internal cell parameter on the direct fundamental band gap $E_{g}$ at the $\Gamma$ point, with quasiparticle corrections of the band gap taken into account, is shown in Fig. 6(b). There is an almost linear variation with a deformation potential $\partial E_{g} / \partial \varepsilon$ of about $-2 \mathrm{eV}$ and an equilibrium band gap $E_{g}^{(0)}=2.108 \mathrm{eV}$. This deformation potential possesses the same sign and is of the same order of magnitude than the volume deformation potential $-9.3 \mathrm{eV}$ of bulk zinc blende $\mathrm{GaP} .^{34}$ 


\section{DISCUSSION AND CONCLUSIONS}

The lattice parameters determined from the Bragg peak positions deviate from what is expected from the bulk $\mathrm{ZB}$ bond length by $\Delta a=-0.31 \%$ and $\Delta c=0.66 \%$, due to the changed stacking sequence of the bilayers and concomitantly changed third next nearest neighbor distance. For several III-V compounds, which are stable as ZB in bulk, for which the WZ unit cell size was investigated so far ( GaAs, InP, InAs, InSb) similar deviations were found for $a$ and $c .^{2,31,32}$ The deviations follow a pseudoexperimental rule published by Lawaetz, ${ }^{35}$ who studied the stability of the WZ phase for a series of $\mathrm{AB}$ compounds and the deviation of the lattice parameters and $c / a$ ratios. He found a systematic deviation with $c / a<\sqrt{8 / 3}$ for compounds with stable WZ phase, while $c / a>\sqrt{8 / 3}$ if $\mathrm{ZB}$ is the stable phase. In Ref. 35 the internal cell parameter $\varepsilon$ was found to have the opposite sign as the $c / a$ deviation. Our measured values are in agreement with this general trend.

To exclude any influence of stacking faults on our determination of the internal parameter $\varepsilon$ we performed simulations to check how the diffracted $\mathrm{x}$-ray intensity depends on the presence of a certain amount of stacking faults. Every stacking fault introduces few atomic layers with cubic stacking, which might influence the unit cell structure of the surrounding WZ material. However, the long defect free segments between the fault planes, which contribute overwhelmingly to the diffracted $\mathrm{X}$-ray signal, consist of the undisturbed WZ phase. Calculations of the diffracted intensity in dependence of the stacking fault density were performed as described in Ref. 36. We assumed an equal amount of stacking faults of different types as described in this reference. We find that for stacking fault densities below $\sim 10$ per $\mu \mathrm{m}$ the deviations of the diffracted intensities from their values for ideal, i. e., defect-free WZ structures are well below $1 \%$ and therefore within the error of the intensity measurements we use in our data analysis. We emphasize that the stacking fault density determined by TEM in our sample is below 1 per $\mu \mathrm{m}$, and thus an order of magnitude smaller. We can therefore exclude any influence of stacking faults on our analysis of $\varepsilon$. Samples with more than 10 stacking faults per $\mu \mathrm{m}$ would, however, render the presented analysis impossible.

For the correct determination of $\varepsilon$ from the intensities of the set of chosen Bragg reflections it is furthermore necessary that the wire length is homogeneous. The homogeneity is important within the spot illuminated by the $\mathrm{x}$-ray beam, varying from 0.01 to $0.014 \mathrm{~mm}^{2}$ for the used Bragg reflections, which is clearly the case. Note that fluctuations from wire to wire do not influence our analysis since within the illuminated spot we average over more than 40000 nanowires. A slight change of wire length for another investigated spot on the sample is not important, as long as the wire length is again homogeneous within this spot.

Using the experimentally determined internal parameter and lattice parameters we find that not only the unit cell but also the bonding tetrahedra are slightly distorted. The bond angles as indicated in Fig. 2(a) are $\alpha=109.81^{\circ}$ and $\beta=109.13^{\circ}$, which correspond to similar (but opposite) deviations as found for WZ GaN. ${ }^{37}$ The bond length along the $c$ direction is $2.358 \AA$ and is therefore slightly shorter than the other bond lengths, which are $2.368 \AA$. Often the deviation from the ideal hexagonal structure is assumed to be driven by the fact that the bond lengths in the tetrahedra stay equal by adjusting the bond angles. For the measured lattice parameters this would mean an expected internal parameter deviation of $\varepsilon=-0.0024$, which is more than twice the measured value. The assumption of equal bond lengths does therefore not hold for WZ GaP. The internal parameter value obtained by DFT calculations tends even more towards a violation from the equal bond length assumption. However, DFT results also underestimate the change in the $c$ lattice parameter, $\Delta c$, which was found to be only $0.27 \%$ instead of the experimental value of $0.66 \%$, while the computed change $\Delta a=-0.30 \%$ is in good agreement with the experimental data.

The magnitude of the internal parameter $\varepsilon$ is directly related to the strength of the spontaneous polarization field $P_{z}$. In bulk wurtzite $P_{z}=\frac{2 e}{V_{\text {cell }}} 4 g \varepsilon$ holds, with the unit cell volume $V_{\text {cell }}$ and an ion charge $4 g$ (see Ref. 16, Supplemental Material), related to the charge-asymmetry coefficient $g=0.371 .^{38}$ With the measured $\varepsilon$-value a polarization $P_{z}=9 \times 10^{-3} \mathrm{C} / \mathrm{m}^{2}$ results. Apart from its sign, this value is only smaller by a factor 2 than that measured for WZ-GaN. ${ }^{17}$ As a consequence, a significant internal electric field is expected for quantum well structures, when zinc blende $\mathrm{GaP}$ is embedded in wurtzite $\mathrm{GaP}$.

In summary we have presented the measurement of the unit cell structure of $\mathrm{GaP}$ in its wurtzite phase including the internal degree of freedom, which has an influence on important material properties like the band gap and the spontaneous polarization field. We used a sample of GaP nanowires grown on a prepatterned (111)B GaP substrate to ensure homogenous growth necessary for our analysis. By synchrotron $\mathrm{x}$-ray diffraction near the Ga- $K$ absorption edge we recorded 3D reciprocal space maps on an ensemble of nanowires. The lattice parameters were found to be $a=3.8419 \pm 0.0004 \AA$ and $c=6.3353 \pm 0.0005 \AA$. For the analysis of the diffracted $\mathrm{X}$-ray intensities we employed the kinematical theory and fit a model of the unit cell structure to the measured data. The Ga-P bond length within the unit cell is determined by the internal cell parameter, which was found to be $u=0.37385 \pm 0.00017$. Using density functional theory the influence of the internal parameter on the direct band gap of $\mathrm{GaP}$ was evaluated and a deformation potential of $-2 \mathrm{eV}$ was found. With the experimentally measured unit cell structure we predict that wurtzite $\mathrm{GaP}$ has a spontaneous polarization of $9 \times 10^{-3} \mathrm{C} / \mathrm{m}^{2}$, which is of magnitude similar to other wurtzite III-V compound materials.

\section{ACKNOWLEDGMENTS}

We acknowledge the help of our local contact for the measurements performed at the synchrotron beamline ID01 ESRF Grenoble (J. Hilhorst) and the financial support of the Austrian Science Fund (FWF P23706-N19) and Austrian Federal Ministry of Science and Research BMWF. D.K. acknowledges the support by the Doctoral Fellowship Programme of the Austrian Academy of Sciences. S.A. acknowledges the Dutch Organization for Scientific Research (NWO-VICI 700.10.441) and the Foundation for Fundamental Research on Matter (FOM) for financial support. 
*dominik.kriegner@gmail.com

${ }^{1}$ A. Mujica and R. J. Needs, Phys. Rev. B 55, 9659 (1997).

${ }^{2}$ M. I. McMahon and R. J. Nelmes, Phys. Rev. Lett. 95, 215505 (2005).

${ }^{3}$ P. Caroff, J. Bolinsson, and J. Johansson, IEEE J. Sel. Top. Quantum Electron. 17, 829 (2011).

${ }^{4}$ R. W. G. Wyckhoff, Crystal Structures, Vol. 1 (Interscience, New York, 1964).

${ }^{5}$ J. Trägårdh, A. I. Persson, J. B. Wagner, D. Hessman, and L. Samuelson, J. Appl. Phys. 101, 123701 (2007).

${ }^{6}$ T. B. Hoang, A. F. Moses, H. L. Zhou, D. L. Dheeraj, B. O. Fimland, and H. Weman, Appl. Phys. Lett. 94, 133105 (2009).

${ }^{7}$ M. Möller, M. M. de Lima, Jr., A. Cantarero, T. Chiaramonte, M. A. Cotta, and F. Iikawa, Nanotechnol. 23, 375704 (2012).

${ }^{8}$ A. Belabbes, C. Panse, J. Furthmüller, and F. Bechstedt, Phys. Rev. B 86, 075208 (2012).

${ }^{9}$ S. Morkötter, S. Funk, M. Liang, M. Döblinger, S. Hertenberger, J. Treu, D. Rudolph, A. Yadav, J. Becker, M. Bichler, G. Scarpa, P. Lugli, I. Zardo, J. J. Finley, G. Abstreiter, and G. Koblmüller, Phys. Rev. B 87, 205303 (2013).

${ }^{10}$ F. Bechstedt and A. Belabbes, J. Phys.: Condens. Matter 25, 273201 (2013).

${ }^{11}$ A. De and C. E. Pryor, Phys. Rev. B 81, 155210 (2010).

${ }^{12}$ S. Assali, I. Zardo, S. Plissard, D. Kriegner, M. A. Verheijen, G. Bauer, A. Meijerink, A. Belabbes, F. Bechstedt, J. E. M. Haverkort, and E. P. A. M. Bakkers, Nano Lett. 13, 1559 (2013).

${ }^{13}$ S. Funk, A. Li, D. Ercolani, M. Gemmi, L. Sorba, and I. Zardo, ACS Nano 7, 1400 (2013).

${ }^{14}$ Lattice mismatch of the WZ phase with respect to the Si (111) surface is $0.04 \%$ and the (111) planes of Si have the same structure as the (000.1) planes of the WZ phase.

${ }^{15}$ R. Resta, J. Phys.: Condens. Matter 22, 3201 (2010).

${ }^{16}$ A. Belabbes, J. Furthmüller, and F. Bechstedt, Phys. Rev. B 87, 035305 (2013).

${ }^{17}$ J. Lähnemann, O. Brandt, U. Jahn, C. Pfüller, C. Roder, P. Dogan, F. Grosse, A. Belabbes, F. Bechstedt, A. Trampert, and L. Geelhaar, Phys. Rev. B 86, 081302 (2012).

${ }^{18}$ G. A. Jeffrey and G. S. Parry, J Chem. Phys. 23, 406 (1955).
${ }^{19}$ G. A. Jeffrey, G. S. Parry, and R. L. Mozzi, J. Chem. Phys. 25, 1024 (1956).

${ }^{20}$ H. Schulz and K. Thiemann, Solid State Commun. 23, 815 (1977).

${ }^{21}$ A. Bauer, J. Kräußlich, L. Dressler, P. Kuschnerus, J. Wolf, K. Goetz, P. Käckell, J. Furthmüller, and F. Bechstedt, Phys. Rev. B 57, 2647 (1998).

${ }^{22}$ F. Bernardini, V. Fiorentini, and D. Vanderbilt, Phys. Rev. B 56, R10024 (1997).

${ }^{23}$ The peak width in XRD corresponds to an average defect free segment length of $>240 \mathrm{~nm}$ slightly smaller than found in TEM, due to the fact that in contrast to TEM also the more defected regions close to the substrate interface contribute in XRD.

${ }^{24}$ U. Pietsch, V. Holy, and T. Baumbach, High-Resolution X-Ray Scattering: From Thin Films to Lateral Nanostructures, Advanced Texts in Physics (Springer, New York, 2004).

${ }^{25}$ B. Ravel and M. Newville, J. Synchrontron Radiat. 12, 537 (2005).

${ }^{26}$ H.-G. Brühl, Krist. Tech. 15, K83 (1980).

${ }^{27}$ E. Sabatke, J. Burge, and D. Sabatke, Appl. Opt. 44, 1360 (2005).

${ }^{28}$ E. Prince and C. Spiegelman, in International Tables for Crystallography, edited by E. Prince and T. R. Welberry, Vol. C (Kluwer Academic, Dordrecht, 2006), Chap. 8.4.

${ }^{29}$ In the fits the $(10 \overline{1} . \overline{5})$ Bragg peak was neglected because the kinematical approximation is not fully valid for this peak.

${ }^{30}$ G. Kresse and J. Furthmüller, Phys. Rev. B 54, 11169 (1996).

${ }^{31}$ D. Kriegner, C. Panse, B. Mandl, K. A. Dick, M. Keplinger, J. M. Persson, P. Caroff, D. Ercolani, L. Sorba, F. Bechstedt, J. Stangl, and G. Bauer, Nano Lett. 11, 1483 (2011).

${ }^{32}$ D. Kriegner, E. Wintersberger, K. Kawaguchi, J. Wallentin, M. T. Borgström, and J. Stangl, Nanotechnol. 22, 425704 (2011).

${ }^{33}$ P. Haas, F. Tran, and P. Blaha, Phys. Rev. B 79, 085104 (2009).

${ }^{34} \mathrm{P}$. Yu and M. Cardona, Fundamentals of Semiconductors (Springer, Berlin, 1996).

${ }^{35}$ P. Lawaetz, Phys. Rev. B 5, 4039 (1972).

${ }^{36}$ M. Barchuk, V. Holý, D. Kriegner, J. Stangl, S. Schwaiger, and F. Scholz, Phys. Rev. B 84, 094113 (2011).

${ }^{37}$ H. Morkoç, Handbook of Nitride Semiconductors and Devices, Vol. 1 (Wiley-VCH, Weinheim, 2008).

${ }^{38}$ A. García and M. L. Cohen, Phys. Rev. B 47, 4215 (1993). 